\title{
Acute Polyneuropathy in an Outpatient Context During the SARS-CoV-2 Pandemic: A Brief Case Serie Report
}

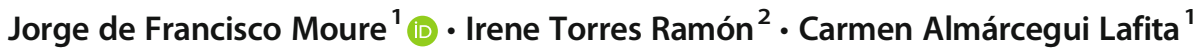

Accepted: 8 March 2021 / Published online: 12 March 2021

(C) The Author(s), under exclusive licence to Springer Nature Switzerland AG 2021

\begin{abstract}
Guillain-Barré syndrome (GBS) is a well-established complication of infectious disease. So it is not surprising that several cases have been described during the actual SARS-CoV-2 infection pandemic. Most of the descriptions are patients suffering a severe GBS in the setting of a severe SARS-CoV-2 infection. We described five patients with mild forms of COVID-19. After 2-4 weeks, these patients develop mild neurological symptoms. The clinical and neurophysiological studies supported a diagnosis of an acute polyneuropathy. Symptoms resolved without specific treatment and primary care physicians managed all patients outpatiently. Mild SARS-CoV-2 infection could associate mild neurological complications too. So patients complaining about mild neurological symptoms, a SARS-CoV-2 infection may be excluded.
\end{abstract}

Keywords COVID-19 $\cdot$ SARS-CoV-2 $\cdot$ Acute polyneuropathy $\cdot$ Guillain-Barré syndrome

\section{Introduction}

Acute polyneuropathy is a well-known complication of systemic infections. A great number of microorganisms have been involved. Generally, it is a severe complication and upon a quarter of patients will need ventilatory support in the course of the disease. However, there are more benign ways. Its pathophysiology is based in molecular mimicry mechanism [1].

In the current pandemic context caused by the novel Coronavirus SARS-CoV-2, several cases of Guillain-Barré syndrome (GBS) have been published. Most of them are severe polyneuropathies in a severe lower respiratory tract infection. Many of these patients required ventilatory support

This article is part of the Topical Collection on COVID-19

Jorge de Francisco Moure

38792jfm@gmail.com

Irene Torres Ramón

mitorres@salud.aragon.es

Carmen Almárcegui Lafita

calmarcegui1@gmail.com

1 Neurophysiology Department, Hospital Universitario Miguel Servet, Paseo Isabel la Católica 1-3, 50009 Zaragoza, Spain

2 Oncology Department, Hospital Clínico Universitario Lozano Blesa, Avenida San Juan Bosco, 15, 50009 Zaragoza, Spain and immunoglobulin treatment [2-9]. Recent studies support the association between GBS and COVID-19 [10, 11], although one analysis did not find this association [12].

We present five cases of an acute peripheral and autonomic nervous system dysfunction in ambulatory patients during the SARS-CoV-2 pandemic. All of these patients had mild upper respiratory tract infection symptoms and all had benign evolutive course. All of these patients received outpatient care and neither required specific treatment.

\section{Case Presentation}

Patient 1: 44-year-old man with a medical history of a chronic ischemic coronary artery disease and hypothyroidism. In the last week of March, he developed a mild upper tract infection (febricula, cough, and anosmia). SARS-CoV-2 was diagnosed by epidemiological criteria but an immunocromatography test made 10 days after the symptoms onset was negative. One week after the initial symptoms, the patient complained about distal paresthesias in lower limbs. In addition, during the ongoing 5 days, paresthesias progressed to the right hand.

His primary care physician treated with paracetamol and the respiratory symptoms resolved in 10 days. But neurological symptoms persisted, so a neurophysiological study was performed 2 months after the initial presentation. At this moment, distal paresthesias persisted in lower limbs. Tactile 
hypoesthesia in feet, arreflexia in lower limbs, and hyporreflexia in upper limbs were still present in the neurological examination. An increment in minimal $\mathrm{F}$ latency and a decrease in its persistency were observed in the neurophysiological study. Signs of a focal neuropathy of median nerve in the carpal tunnel were present too.

Patient 2: A 41-year-old man with type 1 diabetes develops a mild upper tract infection (fever, cough, anosmia) during the first week of April. The patient was symptomatic during 10 days, and his primary care physician treated with paracetamol. SARS-CoV-2 infection was diagnosed by epidemiological criteria and confirmed subsequently with serological methods 15 days after the initial symptom, he complained about mild distal paresthesias in both upper and lower limbs. Because the symptoms did not disappear, the patient consulted again, so his doctor ordered a neurophysiological study.

The study was performed 40 days after the first neurological symptom. On this day, the patient was asymptomatic, but a hyporreflexia was still present in the neurological examination. In the EMG, only an increase in the minimal F-wave latency was observed.

Six months after this initial study, a new EMG was performed and the F-wave latency was normal.

Patient 3: A 49-year-old woman, with a medical history of dyslipemia, consulted in the first week of April to her primary care physician about a mild upper tract infection (fever, headache, arthromyalgias). SARS-CoV-2 infection was diagnosed by epidemiological criteria and confirmed subsequently with serological methods. Five days later, the patient became asymptomatic without treatment. But in the later 15 days, she complained about distal paresthesias in upper and lower extremities. She consulted again with her primary care physician and an EMG was ordered.

One month after the first neurological symptom, the EMG was performed. Although the patient was asymptomatic, hyporreflexia was still present in the neurological examination. The EMG study demonstrated low amplitude sensory nerve action potentials in lower extremities and a focal left median nerve neuropathy in the carpal tunnel. In this case, the F-wave study was normal.

Patient 4: A hypertensive 61-year-old woman suffered a mild upper tract infection (headache, rhinitis, dysgeusia) in June. COVID-19 was confirmed with PCR in nasopharyngeal swab. She consulted to her primary care physician who treated with paracetamol. These symptoms prolonged during 20 days. But when she was asymptomatic, 1 month after the initial symptom, she developed a neuropathic pain in lower extremities. She consulted again with her primary care physician and pregabalin was initiated. Due to the lack of efficacy with this treatment, a neurophysiological study was ordered.

The EMG was performed 45 days after the initial neurological symptom. The neuropathic pain was of lower intensity and the neurological examination was normal, including reflexes. The conventional EMG was normal, but the sympathetic skin response (SSR) was absent. During the exploration, the patient explained us that his son, after a very mild SARSCoV-2 infection, developed a palm and sole hyperhidrosis. So we contacted to this patient (patient 5).

Patient 5: A 29-year-old man, without prior medical history, in a family context of COVID-19, developed a very mild upper tract respiratory infection (febricula, rhinitis, asthenia) that lasted only 1 day. Because of the epidemiological context, a nasopharyngeal swab was performed and the PCR was positive for SARS-CoV-2. Two weeks after this infection, the patient developed a palm and sole hyperhidrosis. One month after this symptom, an EMG was performed. The neurological examination, including reflexes, was normal. Although the conventional tests were normal, the SSR was absent.

\section{Conclusions}

We present these five cases of an acute and mild peripheral and autonomic nervous system dysfunction probably due to a SARS-CoV-2 infection. Although several cases have been published [2-9], most of them are described in patients with severe forms of COVID-19 and the polyneuropathy is also a serious complication that required hospital admission and immunoglobulin treatment.

All of these five patients have mild upper tract respiratory infection. In two patients, this infection was confirmed with a nasopharyngeal swab and a PCR positive for SARS-CoV-2. In the remaining three patients, the diagnostic was made by epidemiological criteria because they were diagnosed in the beginning of the pandemic and PCR was not universally available. But two of them had a serological confirmation of SARS-CoV-2 infection. The last patient did not undergo more explorations.

In the same way, the neurological symptoms were mild too. In three patients, paresthesia and hypo/arreflexia indicate large fiber neuropathy and in the other two (neuropathic pain and hyperhidrosis) suggest small fiber dysfunction. Consistently with these mild symptoms, the neurophysiological studies demonstrated mild or even unspecified changes like prolonged minimal F-wave latency or absent SSR. But in the clinical context and in absence of another explanation, these changes are probably pathological.

All patients were outpatiently treated by their primary care physician and none required a specific treatment. Probably, because of this reason, there were no additional complementary tests, like cerebrospinal fluid analysis or antiganglioside antibodies. So this is an important limitation of the data because we cannot exclude another etiology different from COVID-19. But all patients recovered completely in the following weeks, so an acute and monophasic disease is the most probable. 
The neurological symptoms appeared 2-4 weeks after the upper respiratory tract infection, like classical GBS, so a postinfective physiopathological mechanism is probable [1].

Few patients have been described previously suffering a GBS after a mild or even asymptomatic SARS-CoV-2 infection $[13,14]$. These patients had a more important disease, with muscular weakness and neurophysiological studies showing block conductions, chronodispersion, and prolonged F-wave minimum latency. So these patients were treated with intravenous immunoglobulins and plasma exchange. In contrast, our patients did not have muscular weakness and none needed specific treatment. Moreover, the neurophysiological changes in the studies were milder and in some cases, unspecific. These milder changes correlated with a more benign course of the disease.

In conclusion, we think these cases may contribute to expand the spectrum of SARS-CoV-2 post-infective polyneuropathy in two ways. On one hand, mild SARS$\mathrm{CoV}-2$ infections could cause an acute polyneuropathy too, probably a mild form with subtle or unspecific changes in neurophysiological studies. On the other hand, mild symptoms of an acute peripheral or autonomic nervous system dysfunctions could be due to a SARS-CoV-2 infection, so this possibility could be included in the differential diagnosis.

Supplementary Information The online version contains supplementary material available at https://doi.org/10.1007/s42399-021-00855-x.

Author Contribution Jorge de Francisco had the idea of writing the manuscript, collected data from three of the patients, and wrote the first version of the manuscript. Carmen Almárcegui collected the data for the other two patients and corrected the first version of the manuscript. Irene Torres had the idea of writing the manuscript, helped with the elaboration of the manuscript, and wrote the English version. All of the authors approved the final version.

Data Availability Data submitted in additional file.

Code Availability Not applicable.

\section{Declarations}

Ethical Approval All procedures performed in studies involving human participants were in accordance with the ethical standards of the institutional and/or national research committee and with the 1964 Helsinki declaration and its later amendments or comparable ethical standards.

Consent to Participate Not applicable.

Consent for Publication Not applicable.

Conflict of Interest The authors declare no competing interests.

\section{References}

1. Willison HJ, Jacobs BC, van Doorn PA. Guillain-Barré syndrome. Lancet. 2016;388(10045):717-27. https://doi.org/10.1016/S01406736(16)00339-1.

2. Zhao H, Shen D, Zhou H, Liu J, Chen S. Guillain-Barré syndrome associated with SARS-CoV-2 infection: causality or coincidence? Lancet Neurol. 2020;19(5):383-4. https://doi.org/10.1016/S14744422(20)30109-5.

3. Rahimi K. Guillain-Barre syndrome during COVID-19 pandemic: an overview of the reports. Neurol Sci. 2020;41(11):3149-56. https://doi.org/10.1007/s10072-020-04693-y.

4. Sedaghat Z, Karimi N. Guillain Barre syndrome associated with COVID-19 infection: a case report. J Clin Neurosci. 2020;76: 233-5. https://doi.org/10.1016/j.jocn.2020.04.062.

5. Alberti P, Beretta S, Piatti M, Karantzoulis A, Piatti ML, Santoro P, et al. Guillain-Barré syndrome related to COVID-19 infection. Neurol Neuroimmunol Neuroinflamm. 2020;7:e741. https://doi. org/10.1212/NXI.0000000000000741.

6. Padroni M, Mastrangelo V, Asioli GM, Pavolucci L, AbuRumeileh S, Piscaglia MG, et al. Guillain-Barré syndrome following COVID-19: new infection, old complication? J Neurol. 2020;267:1877-9. https://doi.org/10.1007/s00415-020-09849-6.

7. Caress JB, Castoro RJ, Simmons Z, Scelsa SN, Lewis RA, Ahlawat A, et al. COVID-19-associated Guillain-Barré syndrome: the early pandemic experience. Muscle Nerve. 2020;62(4):485-91. https:// doi.org/10.1002/mus.27024.

8. Camdessanche JP, Morel J, Pozzetto B, Paul S, Tholance Y, Botelho-Nevers E. COVID-19 may induce Guillain-Barre syndrome. Rev Neurol (Paris). 2020;176(6):516-8. https://doi.org/10. 1016/j.neurol.2020.04.003.

9. Abu-Rumeileh S, Abdelhak A, Foschi M, Tumani H, Otto M. Guillain-Barre syndrome spectrum associated with COVID-19: an up-to-date systematic review of 73 cases. J Neurol. 2020;25:1-38. https://doi.org/10.1007/s00415-020-10124-x.

10. Filosto M, Cotti Piccinelli S, Gazzina S, Foresti C, Frigeni B, Servalli MC, et al. Guillain-Barre syndrome and COVID-19: an observational multicentre study from two Italian hotspot regions. J Neurol Neurosurg Psychiatry. 2020;6:jnnp-2020-324837. https:// doi.org/10.1136/jnnp-2020-324837.

11. Fragiel M, Miró Ò, Llorens P, Jiménez S, Piñera P, Burillo G, et al. Incidence, clinical, risk factors and outcomes of Guillain-Barre in Covid-19. Ann Neurol. 2020;9. Online ahead of print. https://doi. org/10.1002/ana.25987.

12. Keddie S, Pakpoor J, Mousele C, Pipis M, Machado PM, Foster M, et al. Epidemiological and cohort study finds no association between COVID-19 and Guillain-Barre syndrome. Brain. 2020;14: awaa433. Online ahead of print. https://doi.org/10.1093/brain/ awaa433.

13. Scheidl E, Diez Canseco D, Hadji-Naumov A. Guillain-Barré syndrome during SARS-CoV-2 pandemic: a case report and review of recent literatura. J Peripher Nerv Syst. 2020;25(2):204-7. https:// doi.org/10.1111/jns.12382.

14. Oguz-Akarsu E, Ozpar R, Mirzayev H, Acet-Ozturk NA, Hakyemez B, Ediger D, et al. Guillain-Barré syndrome in a patient with minimal symptoms of COVID-19 infection. Muscle Nerve. 2020;62(3):E54-7. https://doi.org/10.1002/mus.26992.

Publisher's Note Springer Nature remains neutral with regard to jurisdictional claims in published maps and institutional affiliations. 